\title{
Influencia del riesgo social en la teoría de la mente y funciones ejecutivas de adolescentes colombianos*
}

\section{Influence of Social Risk in the Theory of Mind and Executive Functions of Colombian Teenagers}

Recepción: 02 Mayo 2014 | Aprobación: 24 Enero 2017

María Fernanda UrQuiJo

Universidad del Norte, Colombia

Luis Felipe Zapata ${ }^{\mathrm{a}}$

Universidad del Norte, Colombia

ORCID: http://orcid.org/000-0003-3994-4335

SORAYA LEWIS

Universidad del Norte, Colombia

Wilmar Pineda-Alhucema

Universidad Simón Bolívar, Colombia

Laura Doria Falquez

Universidad del Norte, Colombia

Diana C. LOPERA-PÉrez

Universidad del Norte, Colombia

a Autor de correspondencia. Correo electrónico: lzapata@uninorte.edu.co

Para citar este artículo: Urquijo, M., Zapata, L., Lewis, S., Pineda-Alhucema, W., Falquez, L., \& Lopera-Pérez, D. (2017) Influencia del riesgo social en la teoría de la mente y funciones ejecutivas de adolescentes colombianos. Universitas Psychologica, 16(2), 1-14. https:// doi.org/10.11144/Javeriana.upsy16-2.irst

\section{RESUMEN}

El objetivo del presente estudio fue determinar la influencia del riesgo social en el rendimiento, en tareas de Funciones Ejecutivas (FE) y Teoría de la Mente (ToM) en adolescentes colombianos. Participaron 78 adolescentes (41 en riesgo social y 37 controles) entre los 13 y 16 años de edad seleccionados a través de un muestreo intencional. Se administró el Test de Palabras y Colores de Stroop, el Test de la Pirámide de México y el Test de Falso Paso para evaluar control inhibitorio, planificación y ToM, respectivamente. A pesar de que no se encontraron diferencias significativas en las FE de control inhibitorio y planificación, se encontraron diferencias en el desempeño en algunas dimensiones de la prueba de ToM. Adicionalmente, mientras que al interior del grupo sin riesgo social se observó una relación entre la ToM y las FE evaluadas, al interior del grupo en riesgo social estos procesos no estuvieron relacionados. Se concluye que un entorno de riesgo social parece no afectar las FE de control inhibitorio y planificación o la habilidad para detectar comportamientos inapropiados, pero sí la habilidad para realizar inferencias acerca de los estados mentales de los otros. Asimismo, un entorno de riesgo social es un factor ambiental que parece disociar el proceso de desarrollo de la cognición social y de los procesos ejecutivos en adolescentes, desarrollo que debería darse de manera paralela.

Palabras clave

teoría de la mente; funciones ejecutivas; riesgo social; control inhibitorio

\section{ABSTRACT}

The objective of the present study was to determine the influence of social risk in the performance of Colombian adolescents in ToM and EF 
tasks. The participants were 78 adolescents (41 in social risk and 37 controls) between 13 and 16 years of age. The Stroop Test, the Pyramid of Mexico subtest and the Faux Pas Recognition Test where used to assess inhibitory Control, Planning and ToM in the participants, respectively. Although no significant differences were found in the executive functions of Inhibiting Control and Planning, some differences were found in the performance of the ToM test. Additionally, whereas in the control group a relationship between ToM and executive functions was observed, in the at social risk group these processes were not related. These findings reveal that being at social risk does not affect the Executive Functions of Control and Planning but it does have an effect in the ability to make inferences about the mental states of others. Moreover, being at social risk can be an environmental factor that can dissociate the development process of social cognition and executive functions, which are supposed to occur parallelly. Keywords

theory of mind; executive functions; social risk; inhibitory control

\section{Introducción}

En Colombia existe un gran porcentaje de la población en Riesgo Social (Programa de las Naciones unidas para el Desarrollo [PNUD] y Ministerio de la Protección Social, 2004) y muy poca investigación respecto a cómo este entorno difícil y hostil afecta las habilidades de cognición social y procesos ejecutivos. La cognición social se puede definir como la capacidad o habilidad que permite, tanto a los humanos como a los animales, interpretar de manera adecuada los signos sociales, y consecuentemente, dar una respuesta apropiada a través de los procesos cognitivos superiores que sustentan conductas sociales extremadamente diversas y flexibles (Fiske \& Taylor, 2007; Lieberman, 2013; Suddendorf \& Fletcher-Flinn, 1997).

La cognición social implica diferentes tipos de procesos y habilidades. El reconocimiento de las expresiones faciales emocionales, la comprensión de los gestos y posturas, de la prosodia de la comunicación, la inteligencia emocional, la generación de la empatía y cooperación, así como la Teoría de la mente (ToM) hacen parte de lo que se reconoce como habilidades de cognición social (Tirapu-Ustárroz, Pérez-Sayes, Erekatxo-Bilbao, \& Pelegrín-Valero, 2007; Van der Meer, Groenewold, Nolen, Pijnenborg, \&
Aleman, 2011). Al hablar de ToM, por sus siglas en inglés para Theory of Mind, nos referimos a la capacidad para hacer atribuciones de intenciones, deseos y creencias tanto en los demás como en sí mismo (Leslie, Friedman, \& German, 2004; Premack \& Woodruff, 1978; Tirapu-Ustárroz et al., 2007; Wellman, Cross, \& Watson, 2001). Es decir, es la habilidad para predecir y explicar el comportamiento de las personas en referencia a sus estados mentales. La ToM es considerada un requisito fundamental para nuestra comprensión del mundo social, ya que no podemos conocer con certeza los estados mentales de los otros por el carácter "privado" de la mente. Por este motivo, a los humanos les corresponde generar hipótesis e inferencias acerca de las intenciones, creencias y propósitos de los actos conductuales de los otros (Apperly, Samson, \& Humphreys, 2005; Carpendale \& Lewis, 2010; Pineda Alhucema, Jiménez Figueroa, \& Puentes Rozo, 2012). Así, aunque se opera con cierto grado de incertidumbre respecto a la detección y comprensión de los comportamientos sociales (Brüne, 2004), siempre se trata de comprender al otro para poder ajustarse a un mismo contexto y así establecer relaciones.

\section{ToM y Funciones ejecutivas (FE)}

La puesta en marcha de las habilidades de la ToM supone un cierto grado de control cognitivo que permita realizar la acción con éxito, sobre todo cuando se trata de acciones que implican un contexto social. En este sentido, se puede pensar en las FE justamente como ese controlador cognitivo que pone en marcha las habilidades de cognición social. Pese a que aún no hay una definición definitiva de lo que son las FE, parece haber un consenso en sus funciones: capacidad de planificación de conducta dirigida a una meta, programación de acciones necesarias para alcanzar dicha meta, monitorización de la puesta en marcha del plan para comprobar su ajuste al objetivo y estrategias iniciales, rechazo de la interferencia de estímulos externos que no son relevantes para el plan de acción, 
flexibilidad para la corrección de errores o para la incorporación de conductas nuevas en función de los estímulos del entorno que son relevantes, y la capacidad de reconocer la consecución de los objetivos y finalizar la acción (Arango \& Puerta, 2008; Sanchez-Carpintero \& Narbona, 2001).

No es nuevo proponer una relación entre las FE y la ToM; ya revisiones como las de Papadopoulos, Panayiotou, Spanoudis, y Natsopoulos (2005), proponen varias razones para establecer esta relación. En primer lugar, están las evidencias de que aquellos preescolares que presentan dificultades en tomar perspectivas ajenas, como en la falsa creencia, también tienen claros problemas en el auto-control. Por otro lado, está la activación del lóbulo frontal para ambas funciones (Cañabate, 2014; Saxe, Whitfield-Gabrieli, Scholz, \& Pelphrey, 2009) y por último, el hecho de que en los trastornos del desarrollo hay daños en aspectos similares tanto en la ToM como en las FE. El Trastorno por Déficit de Atención-Hiperactividad constituye un ejemplo de esto último (Miranda-Casas, Baixauli-Fortea, Colomer-Diago, \& RosellóMiranda, 2013; Rumpf, Kamp-Becker, Becker, \& Kauschke, 2012).

Teniendo en cuenta lo anterior, y desde el punto de vista de Blakemore y Choudhury (2006) y Rosselli Cock, Matute, y Jurado (2008), para garantizar el éxito de las relaciones sociales, los humanos necesitan hacer uso de funciones cognitivas de alto orden que provean flexibilidad, control y eficacia en el procesamiento de la información de tipo social. Es de esperar, entonces, que la ToM correlacione positivamente en el curso del desarrollo ontogenético con las funciones ejecutivas (Benson \& Sabbagh, 2009). Según Knobe (2005), y Hale y Tagerflusberg (2003), los individuos que obtienen altas puntuaciones en los test que miden ToM, suelen tener un desempeño positivo en medidas de tipo ejecutivo.

Dentro de las FE, se ha establecido que uno de los aspectos que mayor correlación tiene con la ToM es el Control Inhibitorio y la Capacidad de Planificación (Frye, 2000). Según Carlson, Moses, y Claxton (2004) el Control Inhibitorio es la FE a cargo de suprimir toda la información interna y externa que pueda interferir con la acción a realizar. Esta correlación se basa en que toda interacción social requiere de un filtro que permita que ciertas respuestas fluyan y otras sean detenidas en función de las consecuencias predichas (Leon-Carrion, García-Orza, \& PérezSantamaría, 2004). De igual manera, se ha planteado que la capacidad de planificación se desarrolla en periodos paralelos a los de la ToM, lo que sustenta una relación entre ellas (Barroso \& Carrión, 2002).

No obstante, aunque muchos estudios describen una relación muy cercana entre ToM y FE (Bunge, Dudokovic, Thomason, Vaidya, \& Gabrieli, 2002; Carlson, Claxton, \& Moses, 2015; Muller, Liebermann-Finestone, Carpendale, Hammond, \& Bibok, 2012; Perner \& Lang, 1999), aún no se ha podido establecer la naturaleza de esta relación. Por ejemplo, aún no se ha definido si las FE dependen del funcionamiento de las ToM o viceversa. Alternativamente, los circuitos neuroanatómicos podrían estar superpuestos y entonces no existiría una prevalencia de ninguna de las dos habilidades sobre la otra (Dennis, 2006; Lozano Gutiérrez \& Ostrosky, 2011; Toga, Thompson, \& Sowell, 2006). Por este motivo, todavía se necesitan más estudios y nuevas estrategias para analizar cómo y en qué medida se interconectan las FE y la ToM (Aboulafia-Brakha, Christe, Martory, \& Annoni, 2011; Rothmayr et al., 2011).

\section{Riesgo social}

Entendemos por riesgo social la probabilidad de ocurrencia de un peligro social. La pobreza, entendida como la situación en la que un hogar no logra reunir los recursos necesarios para satisfacer sus necesidades básicas, es un ejemplo de riesgo social. Esto se debe a que el ambiente facilita la adquisición de herramientas sociales: cuando el ambiente no provee los recursos necesarios para el desarrollo social, se habla de un ambiente de riesgo (Feres \& Mancero, 2001).

El concepto de riesgo, sin embargo, implica la posibilidad de que los sujetos no necesariamente sucumban ante tal condición y en cambio, a 
partir de factores protectores, pueden desarrollar nuevas capacidades para afrontar situaciones amenazantes. Tales factores, como los procesos educativos e interpersonales, podrían configurar sus estrategias de afrontamiento de forma positiva y adaptativa frente a presentes y futuros eventos (Aguilera, 2008). Algunos autores han estudiado de qué forma pertenecer a un contexto social de riesgo puede impactar tanto las funciones cognitivas como las habilidades sociales (Arce, Seijo, Fariña, \& MohamedMohand, 2010; Chen, Langer, Raphaelson, \& Matthews, 2004; Choudhury, Blakemore, \& Charman, 2006; Escartí Carbonell et al., 2006). Sin embargo, estos autores han llegado a resultados mixtos en cuanto a la relación entre estas variables. Esto puede deberse a que los estudios han sido realizados en poblaciones con características diferentes (Gogtay et al., 2004).

En Colombia, particularmente, no existen estudios similares replicados en población en riesgo social (Muscatell et al., 2012). Por este motivo, la presente investigación tiene como objetivo determinar la influencia del riesgo social en el rendimiento de tareas de Funciones Ejecutivas (FE) y Teoría de la Mente (ToM) en adolescentes colombianos.

\section{Método y materiales}

\section{Participantes}

La muestra fue seleccionada de forma no probabilística. Participaron 78 sujetos (37 mujeres y 41 hombres), de los cuales 41 correspondían al grupo de riesgo social, y 37 al grupo control. El rango de edad de los participantes se definió entre los 13 y 16 años (media: 14.71; dt: 1.14). La muestra fue extraída de una población de estudiantes de dos colegios de la ciudad de Barranquilla. Para el grupo de adolescentes en riesgo social, se tomó como criterio de inclusión los resultados del Cuestionario de Necesidades Básicas Insatisfechas (NBI) (Feres \& Mancero, 2001) que corroboraran el estado de riesgo del adolescente. Para el grupo control, el criterio de inclusión fue tener características similares al grupo de riesgo social en cuanto a CI, edad, género y escolaridad. El CI de los sujetos fue medido a través de la aplicación del test corto de inteligencia de Reynolds "RIST", (Reynolds \& Kamphaus, 2009) En la tabla 1 se puede observar de qué manera se cumplieron con estos requisitos.

TABLA 1

Características sociodemográficas de la muestra

\begin{tabular}{|c|c|c|c|}
\hline & $\begin{array}{l}\text { Riesgo social } \\
(n=41)\end{array}$ & $\begin{array}{l}\text { Control } \\
(n=37)\end{array}$ & Contraste \\
\hline & $\bar{X}(D t)$ & $\bar{X}(D t)$ & $p$ \\
\hline Edad & $14.59(1.14)$ & $14.84(1.14)$ & NS \\
\hline \multirow[t]{2}{*}{$\mathrm{CI}$} & $69.44(21.11)$ & 75.30 (17.99) & NS \\
\hline & Frecuencia (\%) & Frecuencia (\%) & $p$ \\
\hline Sexo & & & NS \\
\hline$\underline{\text { Masculino }}$ & $24(58.5)$ & $13(35.1)$ & - \\
\hline$\underline{\text { Femenino }}$ & $17(41.5)$ & $24(64.9)$ & - \\
\hline Grado escolar & & & NS \\
\hline$\underline{7}$ & $9(22)$ & $5(13.5)$ & - \\
\hline$\underline{8}$ & $22(53.7)$ & $15(40.5)$ & - \\
\hline$\underline{9}$ & $3(7.3)$ & $2(5.4)$ & - \\
\hline$\underline{10}$ & $6(14.6)$ & $6(16.2)$ & - \\
\hline$\underline{11}$ & $1(2.4)$ & $9(24.3)$ & - \\
\hline
\end{tabular}

NS: No significativa

Fuente: elaboración propia.

\section{Instrumentos}

Para corroborar que los participantes cumplieran con los criterios de inclusión, se aplicaron los siguientes instrumentos:

\section{Cuestionario de Necesidades Básicas Insatisfechas} (NBI)

Este cuestionario (Feres \& Mancero, 2001) fue aplicado para corroborar el estado de riesgo social del adolescente. Fue desarrollado por las Naciones Unidas y la Comisión Económica para América Latina y el Caribe (CEPAL), 
para la identificación de carencias críticas de la población, así como para la caracterización de la pobreza. Los indicadores que utiliza el cuestionario son: a) tipo de vivienda y materiales de construcción de la misma, b) hacinamiento, c) disponibilidad de agua potable, d) acceso a servicios sanitarios, e) asistencia escolar de los menores de edad, y, f) capacidad económica. Este cuestionario ha sido utilizado ampliamente en diferentes estudios con poblaciones latinoamericanas (Núñez, 2013; Torres, Méndez-Fajardo, López-Kleine, GalarzaMolina, \& Oviedo, 2013).

\section{RIST Test de inteligencia breve de Reynolds}

El RIST es un test (Reynolds \& Kamphaus, 2009) de aplicación individual que sirve como una medida breve de la inteligencia. Este test breve tiene una duración aproximada de 15 minutos y fue utilizada en este estudio para controlar la variable de Coeficiente Intelectual en ambas muestras.

Para evaluar las funciones de FE y ToM, se aplicaron los siguientes instrumentos:

\section{El Test de Colores y Palabras STROOP}

Esta prueba mide la FE (Golden, 2001) de Inhibición Cognitiva, ya que requiere de un proceso controlado de una tarea novedosa mientras se debe impedir la interferencia de la lectura, que es un proceso automático (Lezak, Howieson, \& Loring, 2004). La versión del test de Stroop presentado por Golden (2001) consta de tres partes: la primera evalúa la velocidad de lectura de palabras escritas en tinta negra, la segunda la velocidad de denominación de colores de estímulos sin valor semántico y la tercera evalúa la resistencia a la interferencia, ya que la persona debe mencionar el color de la tinta de las palabras presentadas aunque el significado de estas no coincida con el color en el que están impresas. Es decir, el sujeto debe reportar el color de la tinta de la palabra descartando el significado de la misma. El test de Colores y Palabras STROOP se aplica de forma individual y toma cinco minutos aproximadamente para completar. Múltiples autores han utilizado esta prueba para medir la FE en diferentes poblaciones; entre ellos, se encuentran Soar, Chapman, Lavan, Jansari, y Turner (2016) y Sagar et al., (2015).

\section{Sub-test "La pirámide de México" de la Batería de Evaluación Neuropsicológica Infantil (ENI)}

Este sub-test (Matute, Rosselli, Ardila, \& Ostrosky-Solis, 2007) hace parte de la Batería ENI, la cual tiene como objetivo evaluar las características neuropsicológicas de niños y jóvenes en edad escolar. Particularmente, el sub-test "La Pirámide de México", evalúa la efectividad en la capacidad de Planeación y Organización de los sujetos. Para llevar a cabo la prueba, se utilizan tres bloques de tres colores diferentes (verde, blanco y rojo) y de tres tamaños (grande, mediano y pequeño). Una a una, se presentan tarjetas que muestran diferentes modelos de construcción con los bloques. El sujeto debe construir con los bloques el modelo que se le presenta en una tarjeta, teniendo en cuenta que debe realizar el menor número de movimientos posibles de los bloques en el menor tiempo posible. Se obtienen dos puntuaciones: una corresponde al número total de diseños realizados con el número mínimo de movimientos requeridos, y la otra corresponde a si la figura es igual al modelo. La puntuación total correspondiente a cada una de las calificaciones es 11.

Estudios como los de Matute et al., (2008), y Talero-Gutiérrez, Echeverría Palacio, Sánchez Quiñones, Morales Rubio, y Vélez-vanMeerbeke (2015) han utilizado esta prueba para sus investigaciones. Además, en el 2004, RosselliCock et al., llevaron a cabo un estudio normativo para la población colombiana entre los 5 y 16 años de edad.

\section{Test del Falso Paso para adultos}

Conocido también como Faux Pas Recognition Test (Gregory et al., 2002; Stone, Baron-Cohen, \& Knight, 1998), mide la habilidad para detectar 
cuando alguien tuvo un "Falso Paso" o "metedura de pata", es decir, dijo algo sin mala intención pero que resulta inapropiado porque fue o pudo haber sido hiriente para otra persona. La prueba consiste en 10 historias, que contienen un Falso Paso social y 10 historias control que contienen un conflicto menor que no constituye un Falso Paso, agrupadas en orden aleatorio. El evaluador presenta el texto de las historias, una por una en un orden fijo, enfrente del evaluado y las lee en voz alta. Al final, el evaluador hace unas preguntas de memoria para asegurar la comprensión del texto así como las preguntas para medir: Detección, Identificación de lo Inapropiado, Intención, Creencia y Empatía (Gómez, Molina, \& Arana, 2013; Stone, BaronCohen, Calder, Keane, \& Young, 2003).

\section{Procedimiento}

Después de tener la aprobación del comité de ética de la Universidad del Norte, los participantes fueron contactados por medio del Departamento de Trabajo Social de cada institución, en donde se conocen las condiciones sociales de los individuos. Posteriormente, se corroboró el estado de riesgo social a través del cuestionario de Necesidades Básicas Insatisfechas (NBI) (Feres \& Mancero, 2001). Los padres y/o acudientes de los estudiantes aprobaron la participación de los menores a través de una firma sobre el consentimiento informado, que contenía información acerca de los objetivos del proyecto. Para la aplicación de las pruebas, se eligieron horarios durante las pausas de jornada escolar. En casos especiales, las pruebas fueron aplicadas durante clase siempre y cuando la inasistencia no influyera en el desempeño académico de los estudiantes.

En una sola sesión fueron aplicadas todas las pruebas a cada participante. Se mantuvo un solo investigador por cada participante para controlar variables de confusión. El orden de aplicación de los test fue contrabalanceado entre los participantes.

\section{Análisis estadísticos}

Con el fin de evaluar la relación existente entre las FE y la ToM al interior cada uno de los grupos, se realizaron análisis de correlación de Pearson entre cada una de las medidas recogidas. Finalmente, con el fin de determinar si existían diferencias entre los grupos en FE y ToM, se realizaron análisis de comparación de medias de tipo prueba de U de Mann-Whitney para cada una de las medidas recogidas. Todos los análisis fueron realizados utilizando el SPSS 20.

\section{Resultados}

Análisis de comparación de medias

En cuanto al rendimiento en las pruebas, los resultados señalan que solo hay diferencias significativas en dos dimensiones del ToM entre el grupo control y el grupo en riesgo. Tal como se observa en la tabla 2, al realizar una prueba de U de Mann-Whitney se evidencia que el grupo control obtuvo puntuaciones superiores a las del grupo de jóvenes en riesgo social, en la capacidad de identificar la creencia, con un tamaño del efecto pequeño $(\mathrm{r}=0.22)$, mientras que lo contrario ocurrió con la capacidad de identificación de lo inapropiado, también con un tamaño del efecto pequeño $(r=0.22)$. Sin embargo, estas diferencias no se observaron en ninguna de las otras medidas de FE ni en las restantes medidas del TOM. 
TABLA 2

Comparación de medias para las medidas de ToM y FE en ambos grupos

\begin{tabular}{lcll}
\hline & Riesgo Social & Control & \\
& $\overline{\boldsymbol{X}}(\boldsymbol{D} \boldsymbol{t})$ & $\overline{\boldsymbol{X}}(\boldsymbol{D t})$ & \\
\hline Falso paso & & & \\
\hline Detección & $0.819(0.127)$ & $0.839(0.0184)$ & $\mathrm{NS}$ \\
Inapropiado & $0.449(0.557)$ & $0.398(0.095)$ & $<0.05$ \\
Intención & $0.31(0.095)$ & $0.345(0.092)$ & NS \\
Creencia & $0.371(0.091)$ & $0.404(0.092)$ & $<0.05$ \\
Empatía & $0.376(0.083)$ & $0.405(0.096)$ & NS \\
& & & \\
Torre México (Puntuación T) & & & \\
\hline Diseños correctos & $49.85(2.32)$ & $49.19(2.08)$ & NS \\
Movimientos realizados & $48.16(7.88)$ & $48.81(9.77)$ & NS \\
Dis. Mínimo movimientos & $47.74(9.27)$ & $51.78(5.95)$ & NS \\
& & & \\
\hline Stroop (Puntuación T) & & & NS \\
\hline P & & & \\
C & $42.51(7.03)$ & $43.24(6.67)$ & NS \\
\hline & $40.44(7.54)$ & $39.11(7.26)$ & NS \\
\hline
\end{tabular}

NS: No significativa

Fuente: elaboración propia.

Análisis de correlación por cada grupo

Se analizaron las correlaciones entre FE y ToM por cada grupo. En cuanto al grupo control, se encontró relación entre algunas dimensiones de la variable FE y dimensiones de la variable ToM. Como se puede observar en la Tabla 3, estas oscilaron entre 0.333 y 0.392 , lo que corresponde a un tamaño del efecto pequeño. Por el contario, en el grupo de adolescentes en riesgo los análisis de correlación entre las medidas de ToM y FE no mostraron ninguna correlación significativa.
TABLA 3

Correlaciones entre FE y ToM en el grupo control

\begin{tabular}{|c|c|c|c|c|c|}
\hline & \multicolumn{5}{|c|}{ Prueba Falso Paso } \\
\hline Torre México & Detección & Inapropiado & Intención & Creencia & Empatía \\
\hline Diseños correctos & $0.319^{* *}$ & 0.107 & $0.273^{*}$ & $0.252^{*}$ & 0.209 \\
\hline Movimientos realizados & 0.2 & -0.044 & 0.114 & 0.156 & 0.182 \\
\hline $\begin{array}{l}\text { Diseños realizados con el } \\
\text { mínimo de movimientos }\end{array}$ & $0.325^{* *}$ & 0.078 & $0.246^{*}$ & $0.292^{* *}$ & $0.331^{* * *}$ \\
\hline \multicolumn{6}{|l|}{ Stroop } \\
\hline Puntuación T Strop Palabra & 0.1 & -0.003 & 0.025 & 0.08 & $0.228 *$ \\
\hline Puntuación T Stroop Color & 0.135 & -0.047 & 0.179 & 0.093 & 0.102 \\
\hline $\begin{array}{l}\text { Puntuación T Stroop Palabra- } \\
\text { Color }\end{array}$ & 0.103 & -0.013 & 0.088 & 0.117 & 0.167 \\
\hline $\begin{array}{lll}\begin{array}{l}\text { Puntuación } \\
\text { Interferencia }\end{array} & T & \text { Stroop } \\
& & \end{array}$ & 0.062 & 0.04 & 0.07 & 0.091 & 0.114 \\
\hline
\end{tabular}

** La correlación es significativa en el nivel 0.01.

* La correlación es significativa en el nivel 0.05. Fuente: elaboración propia.

\section{Discusión}

El objetivo del presente estudio fue determinar la influencia del riesgo social en el rendimiento de tareas de Funciones Ejecutivas (FE) y Teoría de la Mente (ToM) en adolescentes colombianos con y sin riesgo social. Los resultados arrojados por esta investigación, indican que hay diferencias entre los grupos en la manera cómo perciben los estados mentales de los demás, mas no en su capacidad de Inhibición ni Planificación, y que la ToM solo está correlacionada con los procesos de Inhibición y planificación de las FE en adolescentes que no están en condiciones de riesgo social.

En cuanto a lo primero, los resultados de esta investigación contradicen los resultados de estudios como los de Benarous, Guilé, Consoli, y Cohen (2015) y Luke y Banerjee (2013). Estos autores informan que los niños en condiciones de riesgo social, como ser víctimas de maltrato infantil, presentan bajos desempeños en tareas de funciones cognitivas. De igual forma, se ha encontrado que esto también aplica para los casos de excombatientes con estrés post-traumático (Gómez et al., 2013; Mazza et al., 2012) y personas adultas con trastornos de ansiedad actual asociados a maltrato y abandono infantil.

Esta diferencia puede ser atribuida a que el presente estudio se realizó con adolescentes en situación de riesgo social, entendido como la 
probabilidad de ocurrencia de un peligro social (Aguilera, 2008), y no necesariamente en una población con un diagnóstico clínico. Además, la muestra de este estudio estaba equiparada en cuanto a nivel socieconómico, nivel académico, edad y género. Teniendo esto en cuenta, en principio, la falta de diferencias en cuanto al Control Inhibitorio y a la Planificación pueden explicarse por el hecho de que no se trabajó con población clínica. Esto podría indicar que la variable de riesgo social no es suficiente para afectar las funciones ejecutivas de Control Inhibitorio y Planificación cuando se controlan estas variables, sino que la respuesta individual a estas condiciones puede mediar en sus efectos.

En cuanto a las diferencias encontradas en el desempeño en la tarea de ToM en ambos grupos, podría deberse a que, como lo afirman Portela, Virseda, y Gayubo (2003), la ToM es una habilidad metarrepresentacional en la que el sujeto debe hacer representaciones sobre las representaciones mentales de otros. Esto implica un proceso cognitivo más complejo y de mayor dependencia del ambiente social y al aprendizaje (Miller, 2016; Pears \& Moses, 2003; Rochette \& Bernier, 2016; Wimmer \& Perner, 1983) que explicaría la diferencia negativa en el desempeño del grupo con RS.

Esta alta dependencia de la ToM al ambiente social la hace más sensible a cambios en él, a tal punto que, como lo reportan Mizokawa y Komiya (2014), no siempre se requieren cambios directos en el microsistema social del sujeto para que se afecte, pues alteraciones en los mesosistemas y los macrosistemas también pueden llegar a afectarla (Bronfenbrenner, 1994). De este modo, un ambiente sin adecuadas oportunidades de interacción puede que no ofrezca la estimulación necesaria para crear metarrepresentaciones; por ejemplo, según la hipótesis comunicativa de la ToM, es a través de las interacciones que los niños aprenden a utilizar términos psicológicos para explicar la conducta de otros (Resches, Serrat, Rostan, \& Esteban, 2010; Harris, 2005).

Por otra parte, se ha demostrado que el ambiente puede estimular de forma diferencial algunas dimensiones del desarrollo, y particularmente en contextos de pobreza, la dimensión social podría estar más desarrollada que la cognitiva (De los Reyes-Aragon et al., 2016). Además, es común que las personas en riesgo social mantengan una sensación de peligro constante y desconfianza hacia los demás (Rodríguez, 2015). Estos sentimientos pueden llevar a que tengan más facilidad para reconocer conductas inapropiadas, ya que su supervivencia depende de identificar rápidamente aquellas que los puedan perjudicar. En este sentido, el miedo es una emoción fuerte, capaz incluso de moldear las interacciones sociales de una población (Rebotier, 2011), en donde comportamientos sociales de tipo defensivo son una reacción para la supervivencia.

Otra explicación posible de estas diferencias en la ToM y las no diferencias en la FE halladas en este estudio, podrían ser que la ToM tiene un rol social mucho más directo que las de las FE. Estas últimas, aunque también son mediadas por el ambiente social (Blair, 2016; Mcalister, Schmitter-Edgecombe, \& Lamb, 2016), tienen un funcionamiento más cognitivo ya que en ellas participan otros procesos como la memoria (Craig et al., 2016; Diamond, 2013), el lenguaje (Arango \& Puerta, 2008; Zelazo, Muller, Frye, \& Marcovitch, 2003) la atención (Vilgis, Silk, \& Vance, 2015) entre otras. Mientras tanto, la ToM, es principalmente una función social-cognitiva (Apperly, 2011).

En cuanto a la segunda conclusión, nuestros resultados son un aporte más a aquellos estudios que ya han demostrado la relación entre la función ejecutiva y la teoría de la mente (Aboulafia-Brakha et al., 2011; Arce et al., 2010; Carlson et al., 2015, 2004; Hughes, 1998; Moses \& Tahiroglu, 2010). Sin embargo, en este trabajo se evidenció que, si bien existe una relación entre FE y ToM, está mediada por el ambiente social. La no relación encontrada entre FE y ToM, a su vez, podría explicar por qué personas con el mismo rendimiento en tareas de FE presentan diferencias de rendimiento en tareas de ToM. Esto sugeriría que cuando hay una desconexión entre ambos procesos puede afectarse el rendimiento en las tareas de cognición social. Estos datos invitan a seguir 
indagando sobre la dinámica de esta relación y a considerar variables socioculturales en ella.

En conclusión, es claro que existe una relación entre la teoría de la mente y la función ejecutiva. Sin embargo, parece ser que el ambiente social y, específicamente el riesgo social, tiene cierto grado de influencia en esta relación. Por tanto, para estudios futuros se recomienda seguir investigando en este aspecto empleando más medidas tanto de teoría de la mente como de función ejecutiva de las que se emplearon aquí. Del mismo modo, se recomienda realizar nuevos estudios teniendo en cuenta los antecedentes personales de los adolescentes para identificar posibles episodios de maltrato infantil, trastornos de conducta y estilos de crianza que puedan o hayan podido influir en el actual desempeño en las tareas de ToM y FE.

\section{Agradecimientos}

Esta investigación se realizó con el apoyo de Colciencias en el ámbito del programa Jóvenes Investigadores, convenio 525 del 2011.

\section{Referencias}

Aboulafia-Brakha, T., Christe, B., Martory, M. D., \& Annoni, J. M. (2011). Theory of mind tasks and executive functions: a systematic review of group studies in neurology. Journal of Neuropsychology, 5(1), 39-55.

Aguilera, C. (2008). Análisis del concepto familia en el discurso de un menor en riesgo social. Cyber Humanitatis, 45(15).

Apperly, I. (2011). Mindreaders. The cognitive basis of "Theory of Mind". New York: Psychology Press.

Apperly, I., Samson, D., \& Humphreys, G. W. (2005). Domain-specificity and theory of mind: Evaluating neuropsychological evidence. Trends in Cognitive Sciences, 9(12), 572-577. http://doi.org/10.1016/j.tic s.2005.10.004

Arango, O., \& Puerta, I. (2008). El papel de la función ejecutiva en la conducta, la cognición y la emoción. In D. Pineda (Ed.),
10 años de investigación en neuropsicología (pp. 83-97). Medellín: Universidad de San Buenaventura.

Arce, R., Seijo, D., Fariña, F., \& MohamedMohand, L. (2010). Comportamiento antisocial en menores: Riesgo social y trayectoria natural de desarrollo. Revista Mexicana de Psicología, 27(2), 127.

Barroso, J. M., \& Carrión, J. L. (2002). Funciones ejecutivas: control, planificación y organización del conocimiento. Revista de Psicología General Y Aplicada: Revista de La Federación Española de Asociaciones de Psicología, 55 (1), 27-44.

Benarous, X., Guilé, J.-M., Consoli, A., \& Cohen, D. (2015). A Systematic Review of the Evidence for Impaired Cognitive Theory of Mind in Maltreated Children. Frontiers in Psychiatry, 6(July), 108. doi: http://doi.org/1 $0.3389 /$ fpsyt.2015.00108

Benson, J. E., \& Sabbagh, M. A. (2009). Theory of mind and executive functioning: a developmental neuropsychological approach. In P. D. Zelazo, M. Chandler, y E. Crone (Eds.), Developmental social cognitive neuroscience (pp. 63-80). New York: Psychology Press.

Blair, C. (2016). Executive function and early childhood education. Current Opinion in Behavioral Sciences, 10, 102-107. doi: http: //doi.org/10.1016/j.cobeha.2016.05.009

Blakemore, S. J., \& Choudhury, S. (2006). Development of the adolescent brain: Implications for executive function and social cognition. Journal of Child Psychology and Psychiatry and Allied Disciplines, 47(3-4), 296-312. doi: http://doi.org/10.11 11/j.1469-7610.2006.01611.x

Bronfenbrenner, U. (1994). Ecological models of human development. In T. Husen y T. N. Postlethwaite (Eds.), International encyclopedia of education (2nd ed., pp. 16431647). Oxford: Elsevier.

Brüne, M. (2004). Schizophrenia - An evolutionary enigma? Neuroscience and Biobehavioral Reviews, 28(1), 41-53. doi: http://doi.org/10.1016/j.neubiorev.200 3.10 .002 
Bunge, S., Dudokovic, N., Thomason, M., Vaidya, C., \& Gabrieli, J. D. E. (2002). Immature frontal lobe contributions to cognitive control in children: evidence from fMRI. Neuron, 17, 301-311.

Cañabate, C. (2014). Cognición social. In R. Redolar (Ed.), Neurociencia cognitiva (pp. 693-717). Madrid: Editorial Panamericana.

Carlson, S. M., Claxton, L. J., \& Moses, L. J. (2015). The Relation Between Executive Function and Theory of Mind is More Than Skin Deep. Journal of Cognition and Development, 16(1), 186-197. doi: http://do i.org/10.1080/15248372.2013.824883

Carlson, S. M., Moses, L. J., \& Claxton, L. J. (2004). Individual differences in executive functioning and theory of mind: An investigation of inhibitory control and planning ability. Journal of Experimental Child Psychology, 87 (4), 299-319. doi: http: //doi.org/10.1016/j.jecp.2004.01.002

Carpendale, J. I. M., \& Lewis, C. (2010). The Development of Social Understanding. In The Handbook of Life-Span Development. John Wiley \& Sons, Inc. doi: http://doi.org /10.1002/9780470880166.hlsd001017

Chen, E., Langer, D. A., Raphaelson, Y. E., \& Matthews, K. A. (2004). Socioeconomic status and health in adolescents: The role of stress interpretations. Child Development. doi: http://doi.org/10.1111/j.1467-8624.20 04.00724.x

Choudhury, S., Blakemore, S.-J., \& Charman, T. (2006). Social cognitive development during adolescence. Social Cognitive and Affective Neuroscience, 1(3), 165-74. doi: h ttp://doi.org/10.1093/scan/nsl024

Craig, F., Margari, F., Legrottaglie, A. R., Palumbi, R., de Giambattista, C., \& Margari, L. (2016). A review of executive function deficits in autism spectrum disorder and attention-deficit/hyperactivity disorder. Neuropsychiatric Disease and Treatment, 12, 1191-1202. doi: http://doi.or g/10.2147/NDT.S104620

De los Reyes-Aragón, C., Amar Amar, J., De Castro Correa, A., Lewis Harb, S., Madariaga, C., \& Abello-Llanos, R. (2016).
The care and development of children living in contexts of poverty. Journal of Child and Family Studies, (online first), 1-7.

Dennis, M. (2006). Prefrontal cortex: Typical and atypical development. In The frontal lobes: Development, function and pathology (pp. 128-162). Cambridge: Cambridge University Press.

Diamond, A. (2013). Executive functions. Annual Review of Psychology, 64, 135-68. doi: http://doi.org/10.1146/annurev-psych113011-143750

Escartí Carbonell, A., Gutiérrez, M., Pascual, C., Marín Suelves, D., Martínez Taboada Kutz, C., \& Chacón Flores, Y. (2006). Enseñando responsabilidad personal y social a un grupo de adolescentes de riesgo un estudio «observacional». Revista de Educación, 341, 373-396.

Feres, J. C., \& Mancero, X. (2001). El método de las necesidades básicas insatisfechas (NBI) y sus aplicaciones en América Latina. In Serie estudios estadísticos y prospectivos ( $\mathrm{p}$. 56). Santiago de Chile: Naciones Unidas.

Fiske, S., \& Taylor, S. (2007). Social Cognition: From Brains to Culture. New York: McGraw Hill.

Frye, D. (2000). Theory of mind, domain specificity, and reasoning. In P. Mitchell y K. J. Riggs (Eds.), Children's reasoning and the mind (pp. 149-167). Hove, U.K: Psychology Press.

Gogtay, N., Giedd, J. N., Lusk, L., Hayashi, K. M., Greenstein, D., Vaituzis, a C., ... Thompson, P. M. (2004). Dynamic mapping of human cortical development during childhood through early adulthood. Proceedings of the National Academy of Sciences of the United States of America, 101 (21), 8174-9. doi: http://doi.org/10.107 3/pnas.0402680101

Golden, C. J. (2001). Stroop: Test de colores y palabras (3rd ed.). Madrid: TEA Ediciones.

Gómez, M., Molina, D., \& Arana, M. (2013). Teoría de la mente en un grupo de personas vinculadas al conflicto armado y en proceso de resocialización. Revista Colombiana de Ciencias Sociales, 4(2), 244-257. 
Gregory, C., Lough, S., Stone, V. E., Erzinclioglu, S., Martin, L., Baron-Cohen, S., \& Hodges, J. (2002). Theory of mind in frontotemporal dementia and Alzheimer's disease: Theoretical and practical implications. Brain, 125, 752-764.

Hale, C., \& Tager-flusberg, H. (2003). The Influence of Language on Theory of Mind: A Training Study. Developmental Science, 6(3), 346-359. doi: http://doi.org/10.1111/ 1467-7687.00289

Harris, P. (2005). Conversation, pretense, and Theory of Mind. En J. W. Astington y J. A. Baird (Eds.), Why language matters for theory of mind (pp. 70-83). Nueva York: Oxford University Press.

Hughes, C. (1998). Executive function in preschoolers: Links with theory of mind and verbal ability. British Journal of Developmental Psychology, 16(2), 233-253. doi: http://doi.org/10.1111/j.2044-835X.19 98.tb00921.x

Knobe, J. (2005). Theory of mind and moral cognition: Exploring the connections. Trends in Cognitive Sciences, 9(8), 357-359. doi: http://doi.org/10.1016/j.tics.2005.06.0 11

Leon-Carrion, J., García-Orza, J., y PérezSantamaría, F. J. (2004). Development of the inhibitory component of the executive functions in children and adolescents. The International Journal of Neuroscience, 114(10), 1291-311. http://doi.org/10.1080/ 00207450490476066

Leslie, A. M., Friedman, O., \& German, T. P. (2004). Core mechanisms in "theory of mind." Trends in Cognitive Sciences, 8(12), 528-533. doi: http://doi.org/10.1016/j.tics.2 004.10 .001

Lezak, M.D., Howieson D.B., \& Loring DW. (2004). Neuropsychological assessment. 4a Ed. New York: Oxford University Press.

Lieberman, M. D. (2013). Social: Why our brains are wired to connect. New York: Crown Publishers.

Lozano Gutiérrez, A., \& Ostrosky, F. (2011). Desarrollo de las Funciones Ejecutivas y de la Corteza Prefrontal.
Revista Neuropsicología Neuropsiquiatría, Y Neurociencias, 11(1), 159-172.

Luke, N., \& Banerjee, R. (2013). Differentiated associations between childhood maltreatment experiences and social understanding: A meta-analysis and systematic review. Developmental Review, 33(1), 1-28. doi: http://doi.org/10.1016/j.dr .2012 .10 .001

Matute, E., Chamorro, Y., Inozemtseva, O., Barrios, O., Rosselli, M., \& Ardila, A. (2008). Efecto de la edad en una tarea de planificación y organización (" pirámide de México ") en escolares. Revista de Neurología, 47(2), 61-70.

Matute, E., Rosselli, M., Ardila, A., \& OstroskySolis, F. (2007). Evaluación Neuropsicológica Infantil- ENI. México D.F.: Editorial El Manual Moderno. Universidad de Guadalajara, UNAM.

Mazza, M., Giusti, L., Albanese, A., Mariano, M., Pino, M. C., \& Roncone, R. (2012). Social cognition disorders in military police officers affected by posttraumatic stress disorder after the attack of An-Nasiriyah in Iraq 2006. Psychiatry Research, 198(2), 248252. doi: http://doi.org/10.1016/j.psychres. 2011.11.027

Mcalister, C., Schmitter-Edgecombe, M., \& Lamb, R. (2016). Examination of Variables That May Affect the Relationship Between Cognition and Functional Status in Individuals with Mild Cognitive Impairment: A Meta-Analysis. Archives of Clinical Neuropsychology, 31(2), 123-147. doi: http://doi.org/10.1093/arclin/acv089

Miller, S. (2016). Parenting and Theory of Mind. New York: Oxford University Press.

Miranda-Casas, A., Baixauli-Fortea, I., ColomerDiago, C., \& Roselló-Miranda, B. (2013). Autismo y trastorno por déficit de atención/hiperactividad: convergencias y divergencias en el funcionamiento ejecutivo y la teoría de la mente. Revista de Neurología, 57(1), S177-S184.

Mizokawa, A., \& Komiya, A. (2014). Social ecology and theory of mind. Psychologia, 57(2), 133-151. 
Moses, L. J., \& Tahiroglu, D. (2010). Clarifying the relation between executive function and children's theories of mind. In J. Carpendale, G. Iarocci, U. Müller, B. Sokol, \& A. Young (Eds.), Self- and Social Regulation: Exploring the Relations between Social Interaction, Social Cognition, and the Development of Executive Functions. (pp. 218-231). Oxford: Oxford University Press.

Muller, U., Liebermann-Finestone, D. P., Carpendale, J. I. M., Hammond, S. I., \& Bibok, M. B. (2012). Knowing minds, controlling actions: The developmental relations between theory of mind and executive function from 2 to 4 years of age. Journal of Experimental Child Psychology, 111(2), 331-348. doi: http:// doi.org/10.1016/j.jecp.2011.08.014

Muscatell, K. A., Morelli, S. A., Falk, E. B., Way, B. M., Pfeifer, J. H., Galinsky, A. D., ... Eisenberger, N. I. (2012). Social status modulates neural activity in the mentalizing network. Neurolmage, 60(3), 1771-1777. doi: http://doi.org/10.1016/j.ne uroimage.2012.01.080

Núñez, R. (2013). Pobreza y dificultades para el desarrollo regional. Un diagnóstico de comunidades indígenas Nahuas del municipio de Aquila, Michoacán, desde la perspectiva NBI. INCEPTUM, 8(14), 269284.

Papadopoulos, T. C., Panayiotou, G., Spanoudis, G., \& Natsopoulos, D. (2005). Evidence of poor planning in children with attention deficits. Journal of Abnormal Child Psychology, 33(5), 611-623. doi: http://doi. org/10.1007/s10802-005-6741-8

Pears, K. C., \& Moses, L. J. (2003). Demographics, Parenting, and Theory of Mind in Preschool Children. Social Development, 12 (1), 1-20. doi: http://doi.or g/10.1111/1467-9507.00219

Perner, J., \&Lang, B. (1999). Development of theory of mind and executive control. Trends in Cognitive Sciences, 3(9), 337-344. doi: http://doi.org/10.1016/S1364-6613(99 )01362-5
Pineda Alhucema, W. F., Jiménez Figueroa, G., $\&$ Puentes Rozo, P. (2012). Retrospectiva y prospectiva de la teoría de la mente; avances de investigación en neurociencias. Psicogente, 15(27), 178-197.

Portela, M., Virseda, A., \& Gayubo, L. (2003). Revisión sobre el estudio de la «teoría de la mente» en trastornos generalizados del desarrollo y esquizofrenia. Actas Españolas de Psiquiatría, 31 (6), 339-346.

Premack, D., \& Woodruff, G. (1978). Does the chimpanzee have a theory of mind? Behavioral and Brain Sciences, 1(04), 515526. doi: http://doi.org/10.1017/S0140525 X00076512

Programa de las Naciones unidas para el Desarrollo [PNUD], y Ministerio de la Protección Social. (2004). Investigaciones sobre Desarrollo Social en Colombia. Bogotá.

Rebotier, J. (2011). Politicizing fear of crime and insecurity in Caracas: The manufacturing of a fearful urban metanarrative, Emotion, Space and Society, 4(2),104-112. doi: http://dx.doi.org/10.159 0/S0102-71822013000100017

Resches, M., Serrat, E., Rostan, C., \& Esteban, M. (2010). Lenguaje y teoría de la mente: una aproximación multidimensional. Infancia y Aprendizaje, 33(3), 315-333.

Reynolds, C. R., \& Kamphaus, R. W. (2009). RIAS: Escalas de inteligencia de Reynolds y RIST: Test de Inteligencia breve de Reynolds. (P. Santamaría y I. Fernández, Eds.). Madrid: TEA Ediciones.

Rochette, É., \& Bernier, A. (2016). Parenting and preschoolers' executive functioning: A case of differential susceptibility? . International Journal of Behavioral Development , 40(2), 151-161. doi: http://doi.org/10.1177/016502541455 7370

Rodríguez, M. (2015). La cognición social: ¿una aliada para el postconflicto?, Psicología desde el Caribe, 32(3), 5-7.

Rosselli Cock, M., Matute, E., \& Jurado, M. B. (2008). Las Funciones Ejecutivas a través de la Vida. Revista de Neuropsicología, 
Neuropsiquiatría Y Neurociencias, 8(1), 23 46.

Rosselli-Cock, M., Matute-Villaseñor, E., ArdilaArdila, A., Botero-Gómez, V. E., TangarifeSalazar, G. A., Echeverría-Pulido, S. E., ... Ocampo-Agudelo, P. (2004). Evaluación Neuropsicológica Infantil (ENI): una batería para la evaluación de niños entre 5 y 16 años de edad. Estudio normativo colombiano. Revista de Neurología, 38(8), 720-731.

Rothmayr, C., Sodian, B., Hajak, G., Döhnel, K., Meinhardt, J., \& Sommer, M. (2011). Common and distinct neural networks for false-belief reasoning and inhibitory control. Neurolmage, 56(3), 1705-1713. doi: http://doi.org/10.1016/ j.neuroimage.2010.12.052

Rumpf, A. L., Kamp-Becker, I., Becker, K., \& Kauschke, C. (2012). Narrative competence and internal state language of children with Asperger Syndrome and ADHD. Research in Developmental Disabilities, 33(5), 1395-1407. doi: http://d oi.org/10.1016/j.ridd.2012.03.007

Sagar, K. A., Dahlgren, M. K., Gönenç, A., Racine, M. T., Dreman, M. W., \& Gruber, S. A. (2015). The impact of initiation: Early onset marijuana smokers demonstrate altered Stroop performance and brain activation. Developmental Cognitive Neuroscience, 16, 84-92. doi: http ://doi.org/10.1016/j.dcn.2015.03.003

Sanchez-Carpintero, R., \& Narbona, J. (2001). Revisión conceptual del sistema ejecutivo y su estudio en el niño con trastorno por déficit de atención e hiperactividad. Revista de neurologia, 33(1), 47-53.

Saxe, R. R., Whitfield-Gabrieli, S., Scholz, J., \& Pelphrey, K. A. (2009). Brain regions for perceiving and reasoning about other people in school-aged children. Child Development, 80(4), 1197-1209. doi: http:// doi.org/10.1111/j.1467-8624.2009.01325.x

Soar, K., Chapman, E., Lavan, N., Jansari, A. S., \& Turner, J. J. D. (2016). Investigating the effects of caffeine on executive functions using traditional Stroop and a new ecologically-valid virtual reality task, the Jansari assessment of Executive Functions (JEFC). Appetite, 105, 156-163. doi: http:/ /doi.org/10.1016/j.appet.2016.05.021

Stone, V. E., Baron-Cohen, S., Calder, A., Keane, J., \& Young, A. (2003). Acquired theory of mind impairments in individuals with bilateral amygdala lesions. Neuropsychologia, 41 (2), 209-220. doi: http://doi.org/10.1016/S0028-3932(02 )00151-3

Stone, V. E., Baron-Cohen, S., \& Knight, R. T. (1998). Frontal lobe contributions to theory of mind. Journal of Cognitive Neuroscience, 10, 640-656.

Suddendorf, T., \& Fletcher-Flinn, C. M. (1997). Theory of Mind and the Origin of Divergent Thinking. Journal of Creative Behavior, 31 (3), 169-179. doi: http://doi.or g/10.1002/j.2162-6057.1997.tb00789.x

Talero-Gutiérrez, C., Echeverría Palacio, C. M., Sánchez Quiñones, P., Morales Rubio, G., \& Vélez-van-Meerbeke, A. (2015). Trastorno del espectro autista y función ejecutiva. Acta Neurológica Colombiana, 31(3), 246-252. Recuperado de: http://www.scielo.org.co/scielo.php?scri $\mathrm{pt}=$ sci_arttext\&pid $=$ S0120-87482015000 $300004 \& \operatorname{lng}=$ en $\& n r m=$ iso \& tlng $=$ en

Tirapu-Ustárroz, J., Pérez-Sayes, G., ErekatxoBilbao, M., \& Pelegrín-Valero, C. (2007). ¿Qué es la teoría de la mente? Revista de Neurología, 44(8), 479-489.

Toga, A. W., Thompson, P. M., \& Sowell, E. R. (2006). Mapping brain maturation. Trends in Neurosciences, 29(3), 148-159. doi: http: //doi.org/10.1016/j.tins.2006.01.007

Torres, A., Méndez-Fajardo, S., López-Kleine, L., Galarza-Molina, S., \& Oviedo, N. (2013). Calidad de vida y ciudad: análisis del nivel de desarrollo en Bogotá a través del método de necesidades básicas insatisfechas. Estudios Gerenciales, 29(127), 231-238. doi: http://doi.org/10.1016/j.estge r.2013.05.011

Van der Meer, L., Groenewold, N. A., Nolen, W. A., Pijnenborg, M., \& Aleman, A. (2011). Inhibit yourself and understand the other: 
Neural basis of distinct processes underlying Theory of Mind. NeuroImage, 56(4), 23642374. doi: http://doi.org/10.1016/j.neuroim age.2011.03.053

Vilgis, V., Silk, T. J., \& Vance, A. (2015). Executive function and attention in children and adolescents with depressive disorders: a systematic review. European Child y Adolescent Psychiatry, 24(4), 36584. doi: http://doi.org/10.1007/s00787-015 $-0675-7$

Wellman, H. M., Cross, D., \& Watson, J. (2001). Meta-analysis of theory-of-mind development: the truth about false belief. Child Development, 72 (3), 655-684. doi: ht tp://doi.org/10.1111/1467-8624.00304

Wimmer, H., \& Perner, J. (1983). Beliefs about beliefs: Representation and constraining function of wrong beliefs in young children's understanding of deception. Cognition, 13(1), 103-128. doi: http://doi.org/10.1016 /0010-0277(83)90004-5

Zelazo, P. D., Muller, U., Frye, D., \& Marcovitch, S. (2003). VI. The Development of Executive Function: Cognitive Complexity and Control-Revised. Monographs of the Society for Research in Child Development, 68(3), 93-119. doi: http://doi.org/10.1111/ j.1540-5834.2003.06803007.x

\section{Notas}

* Artículo de investigación. 